\title{
Leitthema
}

Arthroskopie 2013 · 26:99-104

DOI 10.1007/s00142-012-0735-7

Online publiziert: 24. Mai 2013

(c) Springer-Verlag Berlin Heidelberg 2013

Redaktion

P. U. Brucker, München

M. A. Zumstein, Bern
M.O. Schär ${ }^{1,2} \cdot$ S.A. Rodeo ${ }^{2} \cdot$ P. Boileau ${ }^{3} \cdot$ M.A. Zumstein ${ }^{1}$

${ }^{1}$ Schulter, Ellbogen und Sportorthopädie, Universitätsklinik für Orthopädische Chirurgie und Traumatologie, Inselspital, Bern

2 Sports Medicine and Shoulder Service, Hospital for Special Surgery, Cornell University, New York

${ }^{3}$ Department of Orthopaedic Surgery and Sports Traumatology,

L'Archet Hospital II, University of Nice-Sophia-Antipolis

\section{Intraoperative biologische Augmentation an Sehnen und Enthesen}

\begin{abstract}
Sehnen- und Sehenansatzläsionen gehören zu den häufigsten Verletzungen in der Orthopädie. Trotz technischer Weiterentwicklung in der Sehnenrekonstruktionschirurgie besteht postoperativ nach wie vor eine relativ hohe Versagensrate. Ein Grund dafür ist die Tatsache, dass bei der Sehnenansatz- und Sehnenheilung, bedingt durch eine limitierte Regenerationskapazität, Narbengewebe anstelle von normalem Gewebe gebildet wird. Narbengewebe ist dem normalen Gewebe sowohl in funktioneller als auch biomechanischer Sicht unterlegen.
\end{abstract}

Mehrere Strategien zur biologischen Augmentation von Sehnen- und Sehnenansatzläsionen wurden in den letzten Jahren entwickelt und getestet $[2,3,9-12,16,26$, 28]. Einige dieser biologischen Augmentationsstrategien zur Verbesserung der Sehnen-Sehnen- bzw. Knochen-SehnenHeilung wurden bis jetzt erst im Tiermodell untersucht $[3,9-12,16]$. Studien mit biologischen Augmentationen sollten prüfen, ob sich die klinischen Resultate nach Sehnenrekonstruktion dadurch optimieren lassen.

\section{Wachstumsfaktoren}

Wachstumsfaktoren sind Signalmoleküle, welche während der Sehnenheilung die Zellproliferation, Migration, Differenzierung und Matrixsynthese regulie- ren. Sie binden an Zelloberflächenrezeptoren und lösen so intrazelluläre Signalkaskaden aus. Dadurch wird schlussendlich die Desoxyribonukleinsäure(DNS)Transkription und die Produktion von verschiedenen, für die Heilung wichtigen Proteinen induziert. Mehrere Wachstumsfaktoren spielen in der Sehnenheilung eine fördernde Rolle, wie z. B. ,insuline-like growth factor-1" (IGF-1) [22], ,,bone morphogenetic protein“ (BMP-12) [17] sowie "platelet-derived growth factor" (PDGF) [15]. Allerdings zeigte sich, dass die Überexpression von einigen Wachstumsfaktoren, wie z. B. ,transforming growth factor $\beta-1$ “ (TGF $\beta-1)$, zu vermehrter Narbenheilung führt [3]. Ob die Augmentation mit nur einem Wachstumsfaktor das Problem der Sehnen- und Enthesenheilung lösen kann, ist fraglich, da während der Heilung mehrere Wachstumsfaktoren eine Rolle spielen [32].

Autologe plättchenreiche Konzentrate wie plättchenreiches Plasma (PRP) oder plättchenreiches Fibrin (PRF) enthalten mehrere für die Heilung wichtige Wachstumsfaktoren. Bedingt durch die hohe Plättchenkonzentration enthalten diese Konzentrate weit höhere Mengen an Wachstumsfaktoren, als diese bei der physiologischen Wundheilung aus dem Gewebe treten. In den letzten Jahren hat die biologische Augmentation mittels dieser Plättchenkonzentrate zunehmend an Beachtung gewonnen. Der Nutzen solcher Konzentrate zeigte sich in mehreren In-
vitro-Studien [31]. Es konnte u. a. ein positiver Effekt auf die Migration und Proliferation von verschiedenen Zelltypen gezeigt werden [31].

\section{\) Autologe plättchenreiche Konzentrate enthalten wichtige Wachstumsfaktoren}

Die Plättchenkonzentrate unterscheiden sich hinsichtlich ihrer Zusammensetzung und können hierbei in 4 verschiedene Gruppen aufgeteilt werden [7]:

1. Reine plättchenreiche Konzentrate (PPRP)

2. Leukozyten- und plättchenreiche Konzentrate (L-PRP)

3. Reines plättchenreiches Fibrin (PPRF)

4. Leukozyten- und plättchenreiches Fibrin (L-PRF)

Dabei sind 2 verschiedene Parameter zur vollständigen Klassifizierung erforderlich:

a) Während die Gruppen 1 und 3 nur Plättchen enthalten, sind bei den Gruppen 2 und 4 auch Leukozyten enthalten.

b) Die Gruppen 1 und 2 enthalten wenig Fibrin und haben dadurch eine plasmaartige Konsistenz, während die Gruppen 3 und 4 bedingt durch einen hohen Fibringehalt eine Art Matrix enthalten. 

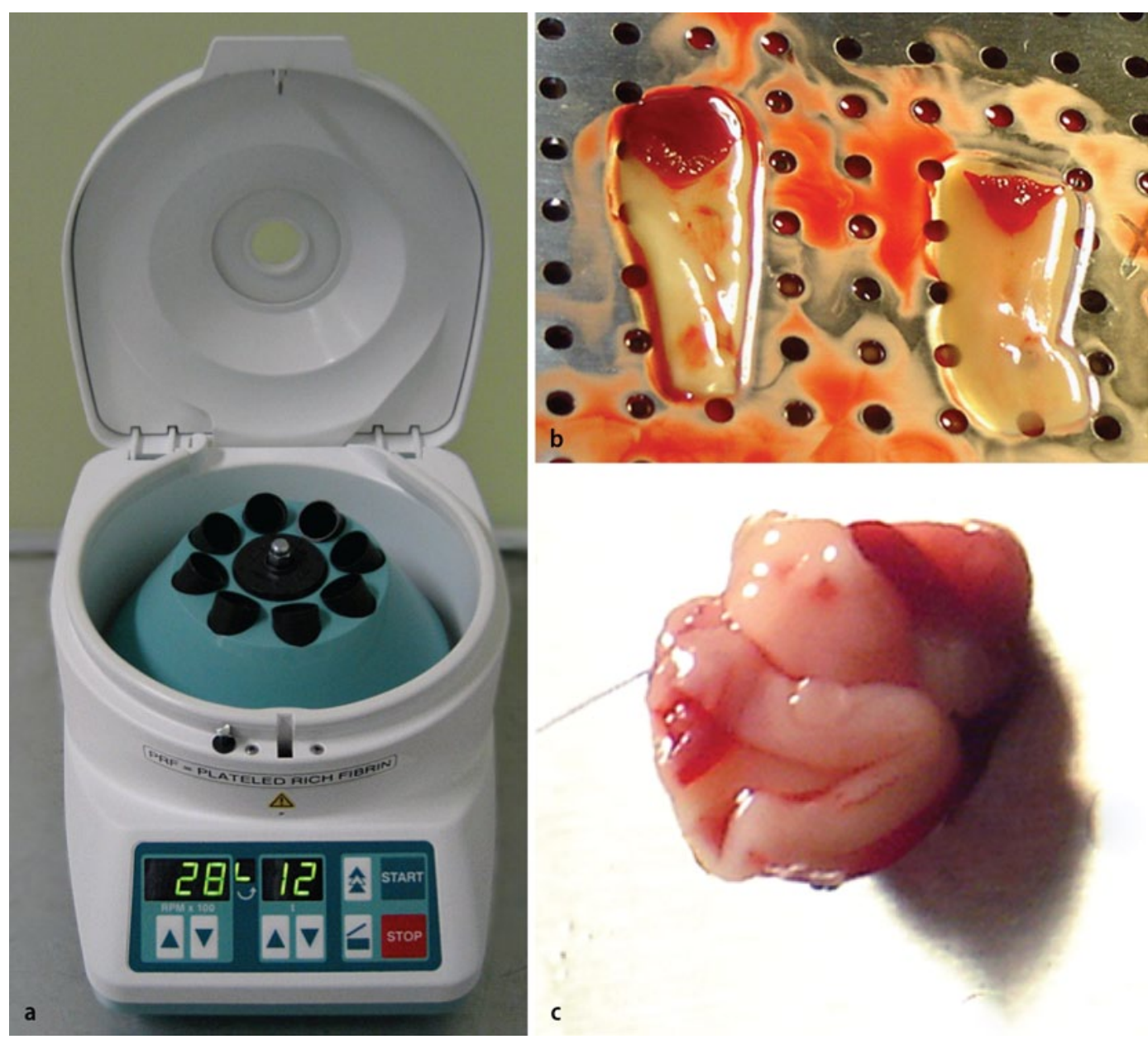

Abb. $1<$ Intraoperative Herstellung eines Konglomerats aus Leukozytenund plättchenreichem Fibrin (L-PRF). a Verwendete Zentrifuge, b intraoperative Herstellung. c Nach Herstellung des L-PRF-Konglomerats werden diese gefaltet und vernäht
Allerdings gibt es trotz wachsendem Interesse für plättchenreiche Konzentrate bisher nur wenige hochqualitative Level-Ioder Level-II-Studien, die den Nutzen dieser Konzentrate untersuchten [6, 26, 28].

\section{Reine plättchenreiche Konzentrate}

In einer prospektiven, randomisierten Studie zeigten Vos et al. [6], dass die Injektion von P-PRP bei einer chronischen Achillodynie zu keiner signifikanten Verbesserung des funktionellen Ergebnisses und der postoperativen Schmerzen im Vergleich zur Natriumchlorid( $\mathrm{NaCl})$-Injektion führt.

\section{Leukozyten- und plättchenreiche Konzentrate}

Randelli et al. [26] führten bei 53 Patienten im Rahmen einer kontrollierten, prospektiv randomisiert durchgeführten Studie eine Rotatorenmanschettenrekonstruktion durch. Bei 26 Patienten wurde die Rekonstruktion zusätzlich mittels L-PRP biologisch augmentiert. Diese Gruppe zeigte in den ersten 30 Tagen postoperativ weniger Schmerzen und nach 3 Monaten einen signifikant besseren Simple-Shoulder-Test (SST), University of California- (UCLA) und ConstantScore (CS). Es fand sich aber kein Unterschied zwischen den beiden Gruppen bezüglich dieser Parameter bzw. Scores nach 6, 12 und 24 Monaten.

\section{Reines plättchenreiches Fibrin}

In einer retrospektiven Fallstudie [29] wurde an 12 Probanden eine Achillessehnenrekonstruktion durchgeführt. Bei 6 der 12 Probanden wurde zusätzlich zur Rekonstruktion P-PRF injiziert. In dieser Gruppe zeigten sich bis 12 Monate postoperativ signifikant bessere funktionelle Ergebnisse mit einer um knapp 8 Wochen verkürzten Rekonvaleszenz in der P-PRFGruppe (14 Wochen in der PRP-Gruppe vs. 21 Wochen in der Kontrollgruppe).
In einer anderen, randomisiert kontrollierten Studie wurde an 79 Patienten eine primäre Rotatorenmanschettenrekonstruktion durchgeführt [28]. Bei der Hälfte der Patienten wurde unmittelbar nach der Rekonstruktion P-PRF appliziert. In dieser Studie zeigte sich kein signifikanter Unterschied zwischen der P-PRF und der Kontrollgruppe bezüglich den klinischen Resultaten [American Shoulder and Elbow Surgeons(ASES)und L'Insalata-Score] und der Heilungsrate $(67 \%$ in der P-PRF-Gruppe vs. $81 \%$ in der Kontrollgruppe) 12 Monate postoperativ. Zudem fand sich kein Unterschied bezüglich der Durchblutung um die Bursa und der Muskel-Sehnen-Einheit. Im Weiteren korrelierten die Thrombozytenzahl und die Heilungsrate nicht miteinander. Es zeigte sich sogar, dass P-PRF ein signifikanter Prädiktor für eine Reruptur 12 Wochen postoperativ in dieser Studie darstellte. Patienten, bei denen die Sehne mit P-PRF augmentiert wurde, wiesen 12 Wochen postoperativ hierbei ein 5,8-fach hö- 
heres Risiko eines Sehnendefektes auf als die Patienten der Kontrollgruppe [28].

Castricini et al. [2] fanden ähnliche Ergebnisse an 88 Patienten und einem 16-Monate-Follow-up. Im CS fand sich kein signifikanter und hinsichtlich der Rerupturrate ein knapp nicht signifikanter Unterschied (P-PRF Gruppe: 1/40, Kontrollgruppe: 4/38; $p=0,07)$ zwischen der P-PRF- und der Kontrollgruppe. Auch die Sehnendicke war in beiden Gruppen nicht signifikant unterschiedlich. Einzig in der Signalintensität der Sehnen zeigte sich in der P-PRF-Gruppe eine erhöhte Signalintensität, was für eine verminderte Sehnenqualität spricht. Eine Korrelation zwischen der Sehnenqualität und dem klinischen Ergebnis fand sich allerdings nicht [2].

\section{Leukozyten- und plättchenreiches Fibrin}

In einer unpublizierten Studie von Zumstein et al. wurde die Rotatorenmanschettenrekonstruktion bei 35 Patienten durchgeführt. Bei allen Patienten wurde eine arthroskopische Doppelreihenrekonstruktion der Rotatorenmanschette durchgeführt, aber nur bei 17 Patienten wurde die Rekonstruktion zusätzlich mit L-PRF augmentiert. Nach Durchführung der Bursektomie und Mobilisation der Rotatorenmanschettensehnen wurde bei retrahierter Rotatorenmanschette ein Release durchgeführt. Der Footprint wurde dann mittels eines Shavers (Acromionizer'; Smith \& Nephew, Andover, MA, USA) angefrischt, um eine gleichmäßig blutende Insertionsstelle zu erhalten. Die medialen absorbierbaren Anker wurden gesetzt und der mediale Anteil der Sehne mit den Ankernähten fixiert, so dass der mediale Anteil der Sehne fest an den medialen Footprint gepresst wurde. So konnte eine spätere Dislokation des LPRF-Konglomerats nach medial verhindert werden. Anschließend wurden gemäß dem PRF-Herstellungsprotokoll pro Patient 4 autologe L-PRF-Konglomerate hergestellt (- Abb. 1a, b). Die L-PRF wurden gefaltet und mittels absorbierbarem Polyglactin 910-Faden (Vicryl, Johnson \& Johnson, Ehicon, Sommerville, NJ, USA) zusammengeheftet (• Abb. 1c). Dieses L-PRF-Konglomerat wurde dann über eine 8,25-mm-Kanüle (Smith \& Nephew, Andover, MA, USA) über das laterale Portal eingebracht und zwischen die Sehne und den dekortizierten Footprint geklemmt (• Abb. 2). AnschlieBend wurde die laterale Ankerreihe gesetzt und der laterale Sehnenanteil daran fixiert (• Abb. 3). Es zeigten sich hierbei keine intra- und postoperative Komplikationen, insbesondere keine Infektionen.

Nach 6 Wochen postoperativ fand sich in der L-PRF-Gruppe eine signifikant erhöhte Durchblutung in der Dopplersonographie verglichen mit der Kontrollgruppe ohne L-PRF-Gabe im Areal, wo die L-PRF-Konglomerate implantiert wurden. Nach 3 Monaten fanden sich in beiden Gruppen je 4 magnetresonanztomographisch nachgewiesene Rerupturen. Zudem zeigten sich nach 3 und 12 Monaten postoperativ keine Unterschiede bezüglich Patientenzufriedenheit, Subjective Shoulder-Value (SSV), Schmerzintensität, SST, CS sowie der Schulterfunktion.

\section{Zellen}

Die Sehnen sind aufgrund ihrer Physiologie sehr zellarm. Die Zellen spielen in der Wundheilung jedoch eine wichtige Rolle. Aus diesem Grund wurde in den letzten Jahren der Einfluss von Augmentationen mittels verschiedener Zellen, wie z. B. mesenchymaler Stammzellen (MSC), untersucht.

\section{1) Sehnen sind aufgrund ihrer Physiologie sehr zellarm}

Mehrere Studien untersuchten, inwieweit MSC die Sehnen-Sehnen- und KnochenSehnen-Heilung verbessern können [10, $11,17,30]$. Es konnte gezeigt werden, dass eine Transduktion von MSC mit verschiedenen Transkriptionsfaktoren, wie z. B. Scleraxis, im Tiermodell zu einer signifikant besseren Knochen-Sehnen-Heilung führt [11]. Scleraxis ist ein Transkriptionsfaktor, der in der Entwicklung der Sehnen im Embryo eine wichtige Rolle spielt [11]. Nach der viralen Transduktion von Stammzellen mit diesem Transkriptionsfaktor differenzieren sich diese Stammzel-
Arthroskopie 2013 · 26:99-104

DOI 10.1007/s00142-012-0735-7

C) Springer-Verlag Berlin Heidelberg 2013

M.O. Schär · S.A. Rodeo · P. Boileau •

M.A. Zumstein

Intraoperative biologische

Augmentation an Sehnen und Enthesen

Zusammenfassung

Trotz technischer Weiterentwicklung in der Sehnenrekonstruktionschirurgie besteht postoperativ nach wie vor eine relativ hohe Rerupturrate. Ein Grund dafür ist die Tatsache, dass bei der Sehnenansatz- und Sehnenheilung, bedingt durch eine limitierte Regenerationskapazität, Narbengewebe anstelle von normalem Gewebe gebildet wird. Dieses Narbengewebe ist sowohl in funktioneller als auch struktureller Sicht dem normalen Gewebe unterlegen. Mehrere Strategien zur biologischen Augmentation von Sehnen- und Sehnenansatzläsionen wurden in den letzten Jahren entwickelt und getestet. Dieser Artikel bespricht die klinische Evidenzlage bezüglich der biologischen Augmentation von Sehnen und Enthesen mittels Wachstumsfaktoren in Form von Plättchenkonzentraten, zellbasierten Therapien bzw. biologischen Sehnengrafts.

\section{Schlüsselwörter}

Narbenheilung · Mesenchymale Stammzellen - Wachstumsfaktoren . Plättchenkonzentrate · Sehnengrafts

\section{Intraoperative biological augmentation of tendons and entheses}

\section{Abstract}

In spite of further developments in surgical techniques for tendon and entheses reconstruction procedures, the retearing rate after tendon and tendon-bone repair remains high. The reason is that instead of native tissue, scar tissue with reduced functional and structural quality forms at the reconstruction site. In order to improve tendon and enthesis healing several therapy strategies have been developed for biological improvement of the repair area. This article provides an overview of the current evidence of augmentation techniques for tendon and enthesis reconstruction using growth factors in the form of platelet concentrates, cell-based therapies and biological tendon grafts.

\section{Keywords}

Scar tissue formation - Mesenchymal stem cells . Growth factors - Platelet concentrates . Tendon grafts 


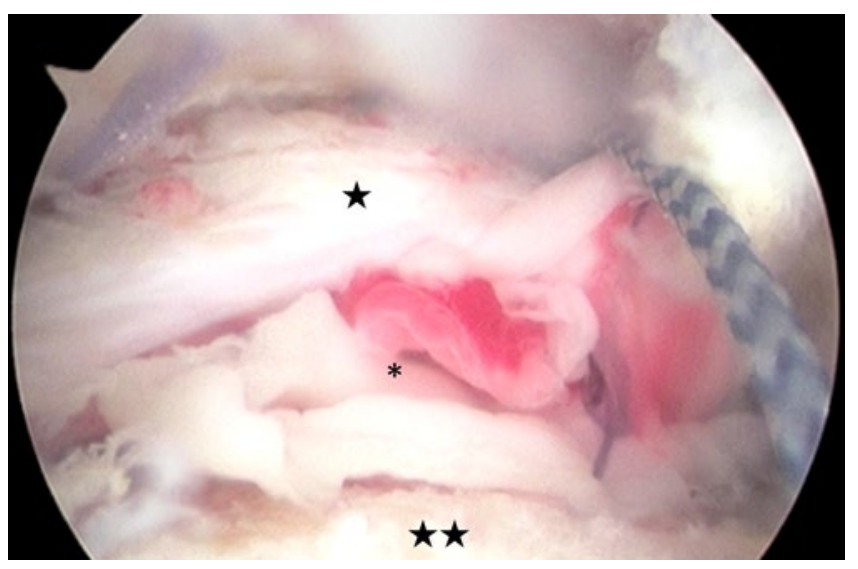

Abb. $2 \triangle$ Das Konglomerat $\left({ }^{*}\right)$ aus Leukozyten- und plättchenreichem Fibrin (L-PRF) wird zwischen die Supraspinatussehne (Stern) und den Knochen (Doppelstern) eingeführt. (Aus [33], mit freundlicher Genehmigung von Elsevier)

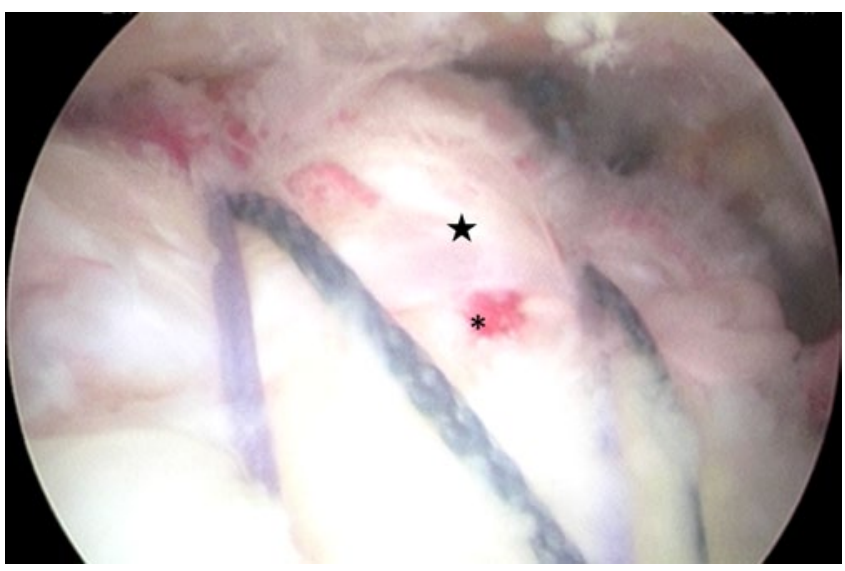

Abb. $3 \Delta$ Arthroskopische Fixation des Konglomerats $\left({ }^{*}\right)$ aus Leukozytenund plättchenreichem Fibrin (L-PRF) zwischen dem Footprint und der Supraspinatussehne (Stern). (Aus [33], mit freundlicher Genehmigung von Elsevier) len zu Sehnenzellen [11]. Eine solche Manipulation der Stammzellen ist aber am Menschen derzeitig nicht erlaubt. Bis heute findet sich nur wenig Evidenz bezüglich dem Nutzen der Augmentation mittels MSC beim Menschen. Mazzocca et al. [19] konnten bei einer arthroskopischen Rotatorenmanschettenrekonstruktion MSC aus dem Knochenmark vom Humerus über den Ankerbohrkanal extrahieren. Diese Stammzellen konnten von $23 \mathrm{~Pa}$ tienten gewonnen und im Labor kultiviert werden. In einer weiteren Studie konnten diese MSC mittels Insulin zu sehnenähnlichen Zellen differenziert werden [20]. Es konnte somit gezeigt werden, dass eine intraoperativ durchgeführte MSC-Extraktion direkt vom Knochenmark des $\mathrm{Hu}$ merus möglich ist und dass die dadurch gewonnenen MSC in sehnenähnliche Zellen differenziert werden können.

Ellera Gomes et al. [8] injizierten bei 14 Patienten unmittelbar nach einer im Mini-open-Verfahren durchgeführten Rotatorenmanschettenrekonstruktion autologe MSC. Nach einem Follow-up von 12 Monaten zeigte sich in allen 14 Patienten eine gute Sehnenintegrität. Nur ein Patient klagte über Kraftverlust und mehr Schmerzen im Vergleich zum präoperativen Zustand.

Die antiinflammatorische Wirkung von MSC auf das umliegende Gewebe konnte in vitro bestätigt werden [1]. Die Injektion von MSC führte in Studien zudem zu einer Verbesserung der Sehnenstruktur $[1,30]$.
Neben den MSC gibt es auch einige nennenswerte Alternativen zur biologischen Augmentation mittels Zellen. In einer randomisierten, kontrollierten Studie an 46 Patienten mit bestehender Patellarsehnentendinitis injizierten Clarke et al. [5] von der Haut stammende sehnenähnliche Zellen („skin-derived tenocyte-like cells"). Diese Hautzellen haben Eigenschaften von Sehnenzellen, sie produzieren u. a. das für die Sehnenheilung wichtige Kollagen. Sechs Monate nach Injektion zeigten Patienten, die eine solche Injektion erhalten hatten, im Vergleich zur Kontrollgruppe, bei der reines Plasma injiziert wurde, bessere klinische Resultate mit einem schnelleren Heilungsverlauf [5].

Auch aus der Plazenta gewonnene pluripotente Zellen und induzierte pluripotente Zellen könnten in der Zukunft möglicherweise zu einer verbesserten Sehnenheilung beitragen [23]. Viele Forscher sowie Kliniker äußern aber Bedenken bezüglich des Gebrauchs am Menschen, da eine potenzielle Ausdifferenzierung in tumorähnliche Zellen nicht ausgeschlossen werden kann [14]. Weitere Studien sind zur Bestimmung des effizientesten Zelltyps, der optimalen Zellzahl und der Trägermatrix nötig.

\section{Sehnengrafts}

Autogene und allogene Sehnengrafts können zur biologischen Augmentation von pathologisch veränderten Sehnen dienen. Sie dienen als Gerüst und helfen so, die reparativen biologischen Prozesse zu beschleunigen. Zudem sind sie Quelle von für die Heilung wichtigen Zellen.

In einer Studie wurde bei 32 Patienten eine Rotatorenmanschettenrekonstruktion durchgeführt und mit einem Sehnenallograft biologisch augmentiert [21]. Alle 15 Patienten wiesen in der 31 Monate postoperativ durchgeführten MRT-Untersuchung eine Reruptur auf. Zudem zeigte sich bei einem Patienten eine Infektion und bei einem Patienten fanden sich Anzeichen einer akuten Abstoßungsreaktion [21].

In einer anderen Studie wurden die bei der Bizepstenotomie entfernten Anteile der Sehne zur Augmentation einer massiven Rotatorenmanschettenrekonstruktion verwendet [27]. Dabei zeigte sich sowohl in der Gruppe, bei der die Rekonstruktion arthroskopisch, sowie in der Gruppe, bei der eine offene Rekonstruktion durchgeführt wurde, eine signifikante Zunahme der Patientenzufriedenheit, des UCLA-Scores, des CS, der Schulterbeweglichkeit, der Kraft, sowie eine korrespondierende Abnahme der Schmerzen im Vergleich zu den präoperativen Werten [27].

\section{Bezüglich des klinischen Outcomes fanden sich keine Unterschiede zwischen der offenen und der arthroskopischen Rekonstruktion.}

Bei einem Vergleich mit herkömmlichen Rekonstruktionsmethoden ohne Sehnengraft weist die Bizepssehneninterposition vergleichbar gute, aber keine besse- 
ren Ergebnisse auf. Dies stellt den Nutzen einer solchen Interposition klar in Frage.

Eine weitere Studie kam zu einem ähnlichen Schluss [4]. Hier zeigte sich zwar eine verminderte Rerupturrate nach Rekonstruktion von großen Rotatorenmanschettenrupturen mit Sehnenaugmentation verglichen mit der Gruppe, bei der keine Augmentation mittels tenotomierter Bizepssehne durchgeführt wurde. Ein Jahr postoperativ fand sich zwischen der Augmentations- und Kontrollgruppe bezüglich Schmerzen, Beweglichkeit und klinischem Ergebnis (UCLA-Score und CS) jedoch kein Unterschied mehr [4].

\section{Indirekte biologische Augmentation}

Eine viel diskutierte Rupturursache ist eine lokale Minderung der Durchblutung in dem Sehnenareal der Supraspinatus- und Infraspinatussehne. In der Vergangenheit wurden deshalb Methoden untersucht, bei denen Sehnen indirekt, z. B. durch die Regulation der Blutzufuhr, beeinflusst werden.

In einer randomisierten doppelverblindeten Studie wurden NitroglycerinPatches bei Patienten mit chronischen Supraspinatussehnenrupturen appliziert [25]. Dabei zeigte sich im Vergleich zu der Placebogruppe ohne Nitroglycerin eine signifikante Verbesserung der Schmerzen nach 24 Wochen, der Kraft nach 12 und 24 Wochen sowie der Beweglichkeit. Nach 6 Monaten waren 46\% der Patienten in der Nitroglyceringruppe und nur 26\% in der Placebogruppe beschwerdefrei, was einen signifikanten Unterschied darstellte.

Weniger gute Ergebnisse zeigten sich in klinischen Studien nach der Anwendung einer extrakorporellen Stoßwellentherapie [13]. Hier war die Gewebeperfusion nach extrakorporeller Stoßwellentherapie bei den Rotatorenmanschettensehnen sogar vermindert [24].

\section{Gegenwärtige Limitationen und Zukunft}

Die zentrale Problematik besteht heute darin, dass nur wenige hochqualitative humane Level-1-Studien den Nutzen der biologischen Augmentation von Sehnen aufzeigen und die vielversprechenden Re- sultate der In-vitro- und Tierstudien bestätigen. Im Weiteren scheint ein besseres Verständnis der Entzündungsprozesse, die mit der Heilung einhergehen und deren Einfluss auf die Heilung essenziell sind, notwendig zu sein.

Weitere Herausforderungen stellen bei der biologischen Augmentation von Sehnen die Evaluation der geeigneten Zellen und/oder Wachstumsfaktoren, deren richtige Konzentration und das richtige Timing dar. Zwar haben Würgler-Hauri et al. [32] den zeitlichen Verlauf der Wachstumsfaktorenausschüttung bei der Sehnenheilung beschrieben. Bei dieser Sehnenheilung wird jedoch nur minderwertiges Narbenmaterial gebildet. Es stellt sich also die Frage, ob eine Imitation des beschriebenen Verlaufs der Wachstumsfaktorenausschüttung der in der Studie beschriebenen Wachstumsfaktorenausschüttung wirklich zu einer verbesserten Sehnenheilung und nicht nur zu noch mehr Narbenbildung führt.

Im Gegensatz dazu wird bei der embryonalen Sehnenheilung kein Narbengewebe gebildet.

\section{> Deshalb wurde versucht, die} Wachstumsfaktorenausschüttung der embryonalen Sehnenheilung zu imitieren.

Manning et al. [18] konnten zeigen, dass die kontinuierliche Ausschüttung von TGF- $\beta 3$, einer TGF- $\beta$-Isoform, die in der embryonalen Sehnenheilung anstelle von TGF- $\beta 1$ ausgeschüttet wird, zu einer verbesserten Sehnenheilung im Tiermodell geführt hat, mit besseren biomechanischen Eigenschaften und weniger Narbenbildung [18].

Aber auch die postoperative Belastung der Sehnen muss weiter untersucht werden, da sie auf die Heilungsrate einen großen Einfluss hat. Letztendlich sind weitere klinische Studien notwendig, um die im Tierversuch gefundenen aussichtsreichen biologischen Augmentationsmethoden zu untersuchen und deren Nutzen für den Menschen zu bestätigen.

\section{Hier steht eine Anzeige.}




\section{Fazit für die Praxis}

- Viele Aspekte der Sehnen- und Enthesenheilung sind heute immer noch unklar.

- Verschiedene Lösungsansätze zur Verbesserung der Sehnen- und Enthesenheilung wurden in den letzten Jahren geprüft.

- Aufgrund der bisherigen Evidenz kann konstatiert werden, dass die Applikation verschiedener Wachstumsfaktoren in Form von Plättchenkonzentraten zur intraoperativen biologischen Augmentation ohne zusätzliche zellbasierte Therapien die klinischen Resultate nicht signifikant verbessert hat.

- Die bisherige Evidenz bezüglich der Augmentation mittels zellbasierten Therapien, z. B. durch Stammzellen, zeigt vielversprechende Ergebnisse, die jedoch durch weitere prospektiv randomisierte klinische Studien bestätigt werden müssen.

- Einen weiteren erfolgversprechenden Lösungsansatz stellt eine Kombination der verschiedenen Augmentationsmethoden dar.

- Bis zur Präsentation klinisch anwendbarer Lösungen werden wohl noch einige Jahre vergehen.

\section{Korrespondenzadresse}

\section{Dr. M.O. Schär}

Schulter, Ellbogen und Sportorthopädie, Universitätsklinik für Orthopädische Chirurgie und Traumatologie, Inselspital

3010 Bern

Schweiz

schaerm@hss.edu

\section{PD Dr. M.A. Zumstein}

Schulter, Ellbogen und Sportorthopädie, Universitätsklinik für Orthopädische Chirurgie und Traumatologie, Inselspital

3010 Bern

Schweiz

matthias.zumstein@insel.ch

Interessenkonflikt. Die korrespondierenden Autoren geben für sich und ihre Koautoren an, dass kein Interessenkonflikt besteht.

\section{Literatur}

1. Aggarwal S, Pittenger MF (2005) Human mesenchymal stem cells modulate allogeneic immune cell responses. Blood 105(4):1815-1822

2. Castricini R, Longo UG, De Benedetto $M$ et al (2011) Platelet-rich plasma augmentation for arthroscopic rotator cuff repair: a randomized controlled trial. Am J Sports Med 39(2):258-265

3. Chang J, Thunder R, Most D et al (2000) Studies in flexor tendon wound healing: neutralizing antibody to TGF-beta 1 increases postoperative range of motion. Plast Reconstr Surg 105(1):148-155

4. Cho NS, Yi JW, Rhee YG (2009) Arthroscopic biceps augmentation for avoiding undue tension in repair of massive rotator cuff tears. Arthroscopy 25(2):183-191

5. Clarke AW, Alyas F, Morris T et al (2011) Skin-derived tenocyte-like cells for the treatment of patellar tendinopathy. Am J Sports Med 39(3):614-623

6. Vos RJ de, Weir A, Schie HT van et al (2010) Platelet-rich plasma injection for chronic Achilles tendinopathy: a randomized controlled trial. JAMA 303(2):144-149

7. Dohan Ehrenfest DM, Rasmusson L, Albrektsson T (2009) Classification of platelet concentrates: from pure platelet-rich plasma (P-PRP) to leucocyteand platelet-rich fibrin (L-PRF). Trends Biotechnol 27(3):158-167

8. Ellera Gomes JL, Silva RC da, Silla LM et al (2012) Conventional rotator cuff repair complemented by the aid of mononuclear autologous stem cells. Knee Surg Sports Traumatol 20(2):373-377

9. Gulotta LV, Kovacevic D, Ehteshami JR et al (2009) Application of bone marrow-derived mesenchymal stem cells in a rotator cuff repair model. Am J Sports Med 37(11):2126-2133

10. Gulotta LV, Kovacevic D, Montgomery S et al (2010) Stem cells genetically modified with the developmental gene MT1-MMP improve regeneration of the supraspinatus tendon-to-bone insertion site. Am J Sports Med 38(7):1429-1437

11. Gulotta LV, Kovacevic D, Packer JD et al (2011) Bone marrow-derived mesenchymal stem cells transduced with scleraxis improve rotator cuff healing in a rat model. Am J Sports Med 39(6):1282-1289

12. Gulotta LV, Kovacevic D, Packer JD et al (2011) Adenoviral-mediated gene transfer of human bone morphogenetic protein-13 does not improve rotator cuff healing in a rat model. Am J Sports Med 39(1):180-187

13. Kim JY, Lee JS, Park CW (2012) Extracorporea shock wave therapy is not useful after arthroscopic rotator cuff repair. Knee Surg Sports Traumato 20(12):2567-2572

14. Knoepfler PS (2009) Deconstructing stem cell tumorigenicity: a roadmap to safe regenerative medicine. Stem Cells 27(5):1050-1056

15. Kobayashi M, Itoi E, Minagawa $\mathrm{H}$ et al (2006) Expression of growth factors in the early phase of supraspinatus tendon healing in rabbits. J Shoulder Elbow Surg 15(3):371

16. Kovacevic D, Fox AJ, Bedi A et al (2011) Calciumphosphate matrix with or without TGF-beta $3 \mathrm{im}$ proves tendon-bone healing after rotator cuff repair. Am J Sports Med 39(4):811-819

17. Lee JY, Zhou Z, Taub PJ et al (2011) BMP-12 treatment of adult mesenchymal stem cells in vitro augments tendon-like tissue formation and defect repair in vivo. PLoS One 6(3):e17531

18. Manning CN, Kim HM, Sakiyama-Elbert S et al (2011) Sustained delivery of transforming growth factor beta three enhances tendon-to-bone healing in a rat model. J Orthop Res 29(7):1099-1105
19. Mazzocca AD, McCarthy MB, Chowaniec DM et a (2010) Rapid isolation of human stem cells (connective tissue progenitor cells) from the proximal humerus during arthroscopic rotator cuff surgery. Am J Sports Med 38(7):1438-1447

20. Mazzocca AD, McCarthy MB, Chowaniec $D$ et al (2011) Bone marrow-derived mesenchymal stem cells obtained during arthroscopic rotator cuff repair surgery show potential for tendon cell differentiation after treatment with insulin. Arthroscopy 27(11):1459-1471

21. Moore DR, Cain EL, Schwartz ML, Clancy WG Jr (2006) Allograft reconstruction for massive, irreparable rotator cuff tears. Am J Sports Med 34(3):392-396

22. Murphy DJ, Nixon AJ (1997) Biochemical and sitespecific effects of insulin-like growth factor I on intrinsic tenocyte activity in equine flexor tendons. Am J Vet Res 58(1):103-109

23. Nagy K, Sung HK, Zhang P et al (2011) Induced pluripotent stem cell lines derived from equine fibroblasts. Stem Cell Rev 7(3):693-702

24. Notarnicola A, Moretti L, Tafuri S et al (2011) Reduced local perfusion after shock wave treatment of rotator cuff tendinopathy. Ultrasound Med Biol 37(3):417-425

25. Paoloni JA, Appleyard RC, Nelson J, Murrell GA (2005) Topical glyceryl trinitrate application in the treatment of chronic supraspinatus tendinopathy: a randomized, double-blinded, placebo-controlled clinical trial. Am J Sports Med 33(6):806-813

26. Randelli $P$, Arrigoni P, Ragone V et al (2011) Platelet rich plasma in arthroscopic rotator cuff repair: a prospective RCT study, 2-year follow-up. J Shoulder Elbow Surg 20(4):518-528

27. Rhee YG, Cho NS, Lim CT et al (2008) Bridging the gap in immobile massive rotator cuff tears: augmentation using the tenotomized biceps. Am J Sports Med 36(8):1511-1518

28. Rodeo SA, Delos D, Williams RJ et al (2012) The effect of platelet-rich fibrin matrix on rotator cuff tendon healing: a prospective, randomized clinical study. Am J Sports Med 40(6):1234-1241

29. Sanchez M, Anitua E, Azofra J et al (2007) Comparison of surgically repaired Achilles tendon tears using platelet-rich fibrin matrices. Am J Sports Med 35(2):245-251

30. Schnabel LV, Lynch ME, Meulen MC van der et al (2009) Mesenchymal stem cells and insulin-like growth factor-I gene-enhanced mesenchymal stem cells improve structural aspects of healing in equine flexor digitorum superficialis tendons. J Orthop Res 27(10):1392-1398

31. Visser LC, Arnoczky SP, Caballero O, Egerbacher M (2010) Platelet-rich fibrin constructs elute higher concentrations of transforming growth factor-beta1 and increase tendon cell proliferation over time when compared to blood clots: a comparative in vitro analysis. Vet Surg 39(7):811-817

32. Wurgler-Hauri CC, Dourte LM, Baradet TC et al (2007) Temporal expression of 8 growth factors in tendon-to-bone healing in a rat supraspinatus model. J Shoulder Elbow Surg 16(5, Supplement 1):S198

33. Zumstein MA, Bielicki T, Dohan Ehrenfest DM (2011) The future of platelet concentrates in sports medicine: platelet-rich plasma, platelet-rich fibrin, and the impact of scaffolds and cells on the longterm delivery of growth factors. Oper Tech Sports Med 19(3):190-197 